\title{
LncRNA TUG1 serves an important role in hypoxia-induced myocardial cell injury by regulating the miR-145-5p-Binp3 axis
}

\author{
ZHONGWEI WU, SHENGJI ZHAO, CHUNFU LI and CHAOQUAN LIU \\ Department of Cardiology, Hainan Western Central Hospital, Danzhou, Hainan 571700, P.R. China
}

Received July 27, 2017; Accepted November 6, 2017

DOI: $10.3892 / \mathrm{mmr} .2017 .8116$

\begin{abstract}
The aim of the present study was to investigate the function of long non-coding RNA TUG1 in hypoxia-induced myocardial cell injury and to explore the potential molecular mechanisms. The cardiomyocyte cell line H9c2 was cultured under hypoxic and normoxic conditions. TUG1 expression under hypoxic conditions was then detected. The effects of TUG1 overexpression on viability, apoptosis, migration and invasion were assayed. In addition, the microRNA (miR)-145-5p expression was detected. Following H9c2 cell transfection with miR-145-5p mimics, the H9c2 cell viability, apoptosis, migration and invasion were also detected. Additionally, the target gene of miR-145-5p was assayed by Luciferase reporter assay. The protein expressions of Wnt-3a, Wnt5a, and $\beta$-catenin in $\mathrm{H} 9 \mathrm{c} 2$ cells under hypoxic conditions were also determined. The results revealed that hypoxia induced injury in $\mathrm{H} 9 \mathrm{c} 2$ cells, including inhibiting cell viability, migration and invasion, and promoting cell apoptosis. Overexpression of TUG1 aggravated hypoxia-induced injury in H9c2 cells. In addition, miR-145-5p was negatively regulated by TUG1, and TUG1 overexpression aggravated hypoxia-induced injury via the downregulation of miR-145-5p. Furthermore, B-cell lymphoma 2 interacting protein 3 (Bnip3) was a target of miR-145-5p, and overexpression of Bnip3 aggravated hypoxia-induced cell injury by activating $\mathrm{Wnt} / \beta$-catenin signaling pathways in $\mathrm{H} 9 \mathrm{c} 2$ cells. In conclusion, overexpression of TUG1 aggravated hypoxia-induced injury in cardiomyocytes by regulating the miR-145-5p-Binp3 axis. Activation of the Wnt/ $\beta$-catenin signaling pathway may be a key mechanism to mediate the role of TUG1 in regulating hypoxia-induced myocardial injury. TUG1 may be an effective diagnostic marker and therapeutic target for myocardial ischemia.
\end{abstract}

Correspondence to: Professor Zhongwei Wu, Department of Cardiology, Hainan Western Central Hospital, 2 East Fubo Road, Nada, Danzhou, Hainan 571700, P.R. China

E-mail: zhongweiwu02@sina.com

Key words: myocardial ischemia, hypoxia, long non-coding RNA taurine upregulated 1 (non-protein coding), microRNA-145-5p, B-cell lymphoma 2 interacting protein 3

\section{Introduction}

Myocardial ischemia is a common cause of morbidity and mortality in the world (1). It is well known that well-differentiated tissues including heart require large amounts of oxygen to support their specialized functions. When oxygen is in short supply, the oxidative phosphorylation of mitochondria stops rapidly, which results in a resultant loss of the major source of ATP production for energy metabolism and subsequently ischemia $(2,3)$. Myocardial ischemia can cause a characteristic pattern of ultrastructural and metabolic changes, leading to irreversible damage to the myocardium $(4,5)$. Presently, the mechanisms of myocardial ischemic injury are still needed to be explored.

Long non-coding RNAs (lncRNAs) are a set of RNAs longer than $200 \mathrm{nt}$, which involve in lots of cellular processes, such as genomic imprinting, chromatin modification and RNA alternative splice (6). In addition, IncRNAs are associated with many human diseases (7-9). Recently, a growing number of studies focus on the role of IncRNAs in cardiac diseases. Several lncRNAs have been detected in cardiomyocytes and are suggested to be involved in heart development $(10,11)$. LncRNA TUG1 is highly conserved in mammals but it is not reported in other vertebrates (12). Previous studies have shown that TUG1 is implicated in many cancers, affecting apoptosis and proliferation of tumor cells $(13,14)$. However, to our best knowledge, there is no study concerning the function of TUG1 in regulating myocardial ischemic injury.

Therefore, to explore the role and regulatory mechanism of TUG1 in regulating myocardial ischemic injury, the present study established a cell model of myocardial injury through treating cardiomyocytes with hypoxia. Then the expression level of TUG1 in hypoxia-induced myocardial injury model was detected. Furthermore, the relationship between dysregulated expression of TUG1 and myocardial injury were explored, as well as the potential molecular mechanisms of TUG1 in regulating myocardial injury. This study aimed to provide new theoretical explanation for the mechanism of myocardial injury.

\section{Materials and methods}

Cell culture and treatment. The cardiomyocytes cell line H9c2 was purchased from Sigma-Aldrich (Merck KGaA, Darmstadt, Germany), and cultured in Dulbecco's modified 
Eagle's medium (Thermo Fisher Scientific, Inc., Waltham, MA, USA) at $37^{\circ} \mathrm{C}$ in an incubator with $5 \% \mathrm{CO}_{2}$. The culture medium was supplemented with $10 \%$ fetal bovine serum (FBS), 1\% Penicillin/Streptomycin (100 U/ml:100 mg/ml) and 1\% GlutaMAX (Thermo Fisher Scientific, Inc.), and was changed every other day. The H9c2 cells were cultured under the hypoxia $\left(3 \% \mathrm{O}_{2}\right)$ and normoxia $\left(21 \% \mathrm{O}_{2}\right)$ conditions, respectively.

Cell transfection. Short-hairpin (sh)RNA directed against TUG1 was ligated into the plasmid of U6/GFP/Neo (GenePharma, Shanghai, China), which was called sh-TUG1. TUG1 was ligated into the pcDNA3.1, which was referred as to pc-TUG1. To analyze the functions of Bcl2/adenovirus E1B 19 kDa-interacting protein 3 (Bnip3), the full-length Bnip3 sequences and shRNA directed against Bnip3 were respectively ligated into plasmids of pEX-2 and U6/GFP/Neo (GenePharma), referring as to pEX-Bnip3 and si-Bnip3. Cells transfection was then performed using Lipofectamine 3000 reagent (Thermo Fisher Scientific, Inc.) according to the manufacturer's instructions. The plasmid that carried a non-targeting sequence was used as negative control (NC) of sh-TUG1 and si-Bnip3. The stably transfected cells were selected through the culture medium containing $0.5 \mathrm{mg} / \mathrm{ml} \mathrm{G} 418$ (Sigma-Aldrich; Merck KGaA), and G418-resistant cell clones were established after about 4 weeks. miR-145-5p mimics, miR-145-5p inhibitors, and NC were synthesized (Thermo Fisher Scientific, Inc.) and then transfected into cells. Cells were harvested after $72 \mathrm{~h}$ of transfection.

$R T-q P C R$. Total RNA was extracted from cells using Trizol reagent (Thermo Fisher Scientific, Inc.). Real-Time PCR analysis was performed to detect the expression level of TUG1 using One Step SYBR ${ }^{\circledR}$ PrimeScript ${ }^{\circledR}$ PLUS RT-RNA PCR Kit (TaKaRa Biotechnology). Bnip3 expression was detected with RNA PCR kit (AMV) Ver.3.0 (Takara Biotechnology Co., Ltd., Dalian, China). GAPDH was used as the internal control. The expression level of miR-145-5p was determined using the Taqman MicroRNA Reverse Transcription Kit and Taqman Universal Master Mix II with the TaqMan MicroRNA Assay of miR-145-5p and U6 (Applied Biosystems; Thermo Fisher Scientific, Inc.). U6 was used for normalizing the expression of miR-145-5p. Fold-changes were calculated according to cycle quantitation $(\mathrm{Cq})$ values with $2^{-\Delta \Delta \mathrm{Cq}}$ method.

Cell viability assay. Total $1 \times 10^{5}$ cells were seeded into $60-\mathrm{mm}$ dishes in duplicate. At the indicated time periods, Cells were washed and the living cells were determined using trypan blue exclusion.

Apoptosis assay. Cells were washed with phosphate-buffer saline (PBS) and then fixed in $70 \%$ ethanol. Afterwards, cells were stained with propidium iodide (PI)/fluorescein isothiocyanate (FITC)-Annexin V in the presence of $50 \mu \mathrm{g} / \mathrm{ml}$ RNase A (Sigma-Aldrich; Merck KGaA). Then cells were incubated in the dark for $1 \mathrm{~h}$ at $25^{\circ} \mathrm{C}$. Flow cytometry analysis was performed using a FACS can (Beckman Coulter, Inc., Brea, CA, USA). The data were analyzed using the FlowJo software.

Cell migration and invasion assays. Cell migration was detected using a modified two-chamber migration assay (pore size,
$8 \mathrm{~mm}$ ). Cells suspended in $200 \mathrm{ml}$ serum-free medium were seeded on the upper compartment of 24-well Transwell culture chamber, and $600 \mathrm{ml}$ complete medium was added to the lower compartment. After incubation at $37^{\circ} \mathrm{C}$, cells were then fixed with methanol. On the upper surface of the chamber, the non-traversed cells were removed with a cotton swab. The traversed cells were stained with crystal violet and counted microscopically.

The invasion behavior was detected with 24-well Millicell Hanging Cell Culture inserts with $8 \mathrm{~mm}$ PET membranes (EMD Millipore, Billerica, MA, USA). Total 5.0 $\times 10^{4}$ cells suspended in $200 \mu \mathrm{l}$ serum-free dulbecco's modified eagle medium were seeded onto BD BioCoat ${ }^{\mathrm{TM}}$ Matrigel TM Invasion Chambers (BD Biosciences, Franklin Lakes, NJ, USA). Complete medium containing $10 \%$ FBS was added to the lower chamber. The invasion chambers were incubated at $37^{\circ} \mathrm{C}$ for $48 \mathrm{~h}$ with $5 \% \mathrm{CO}_{2}$. After removing the non-invading cells, the invading cells were fixed with $100 \%$ methanol and stained with crystal violet solution. Finally, cells were counted microscopically.

Luciferase reporter assay. Fragment from Bnip3 that contained the predicted miR-145-5p binding site was amplified via PCR, which were then cloned into a pmirGlO Dual-luciferase miRNA Target Expression Vector (Promega Corporation, Madison, WI, USA) to construct the reporter vector Bnip3-wild-type (Bnip3-wt). Subsequently, the reporter vectors and miR-145-5p mimics were co-transfected into HEK 293 T cells. The luciferase activity was determined based on the Dual-Luciferase Reporter Assay System (Promega Corporation).

Western blot analysis. Protein was extracted using RIPA lysis buffer (Beyotime Biotechnology, Shanghai, China) that was supplemented with protease inhibitors (Roche, Guangzhou, China). The protein extracts were quantified with the $\mathrm{BCA}^{\mathrm{TM}}$ Protein Assay kit (Pierce; Thermo Fisher Scientific, Inc.). According to the manufacturer's instructions, the western blot system was established using a Bio-Rad Bis-Tris Gel system. Primary antibodies (at a dilution of 1:1,000) were prepared in $5 \%$ blocking buffer. After incubation with primary antibodies at $4^{\circ} \mathrm{C}$ overnight, secondary antibodies marked by horseradish peroxidase were used to incubate the polyvinylidene difluoride (PVDF) membrane at room temperature (approximately $25^{\circ} \mathrm{C}$ ) for $1 \mathrm{~h}$. The membranes carried blots and antibodies were then transferred into the Bio-Rad ChemiDoc ${ }^{\mathrm{TM}}$ XRS system, and $200 \mu 1$ Immobilon Western Chemiluminescent HRP Substrate (EMD Millipore) was added to cover the membrane surface. The intensity of the bands was quantified with Image Lab ${ }^{\mathrm{TM}}$ Software (Bio-Rad, Shanghai, China).

Statistical analysis. The results were presented as mean \pm standard deviation. Statistical analyses were performed using Graphpad 6.0. The P-values were calculated using a one-way analysis of variance (ANOVA). $\mathrm{P}<0.05$ was considered to indicate a statistically significant difference.

\section{Results}

Hypoxia induces hypoxia injury in $\mathrm{H} 9 \mathrm{c} 2$ cells. The effects of hypoxia on $\mathrm{H} 9 \mathrm{c} 2$ cells were evaluated by determination of the 

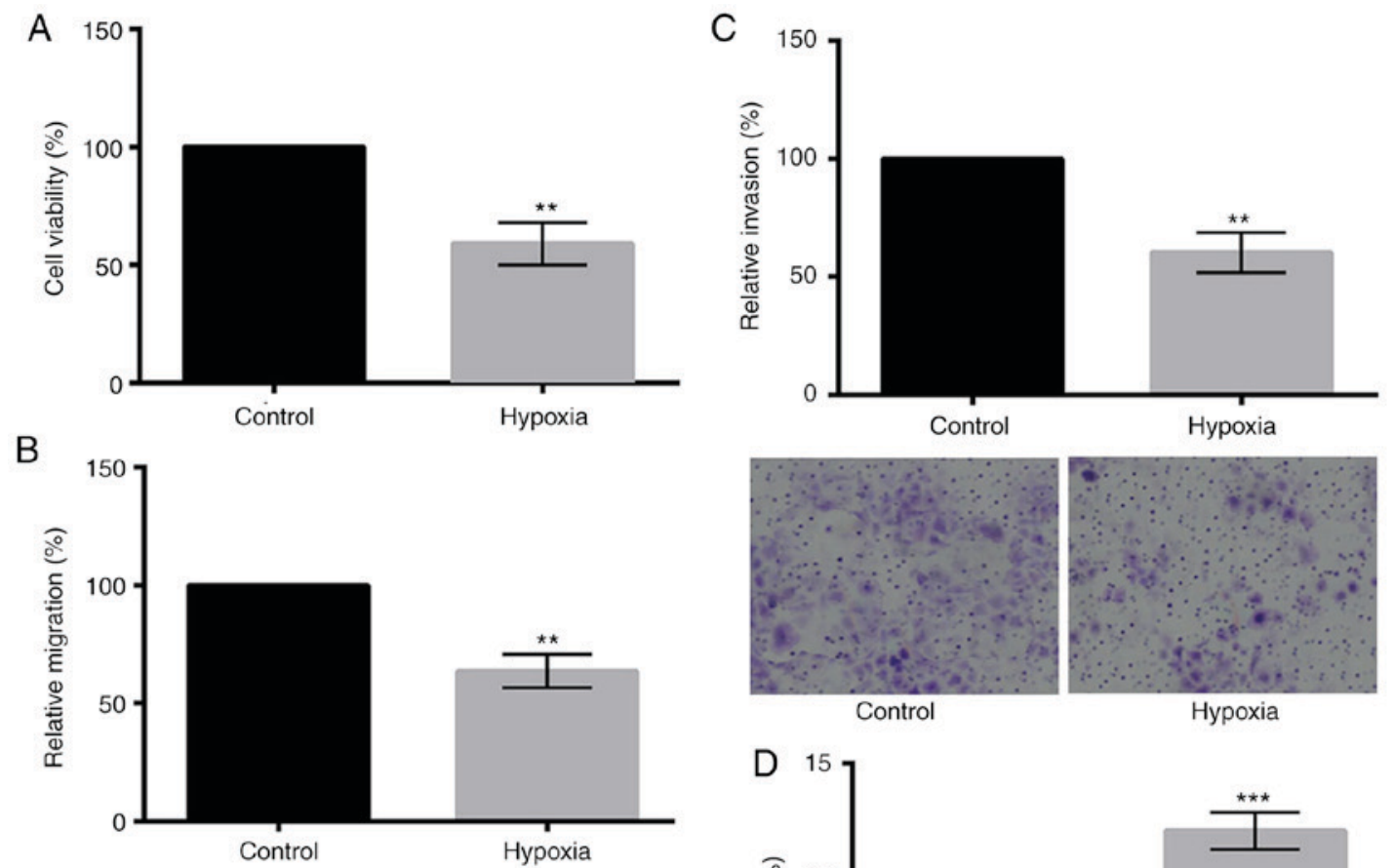

Control

Hypoxia
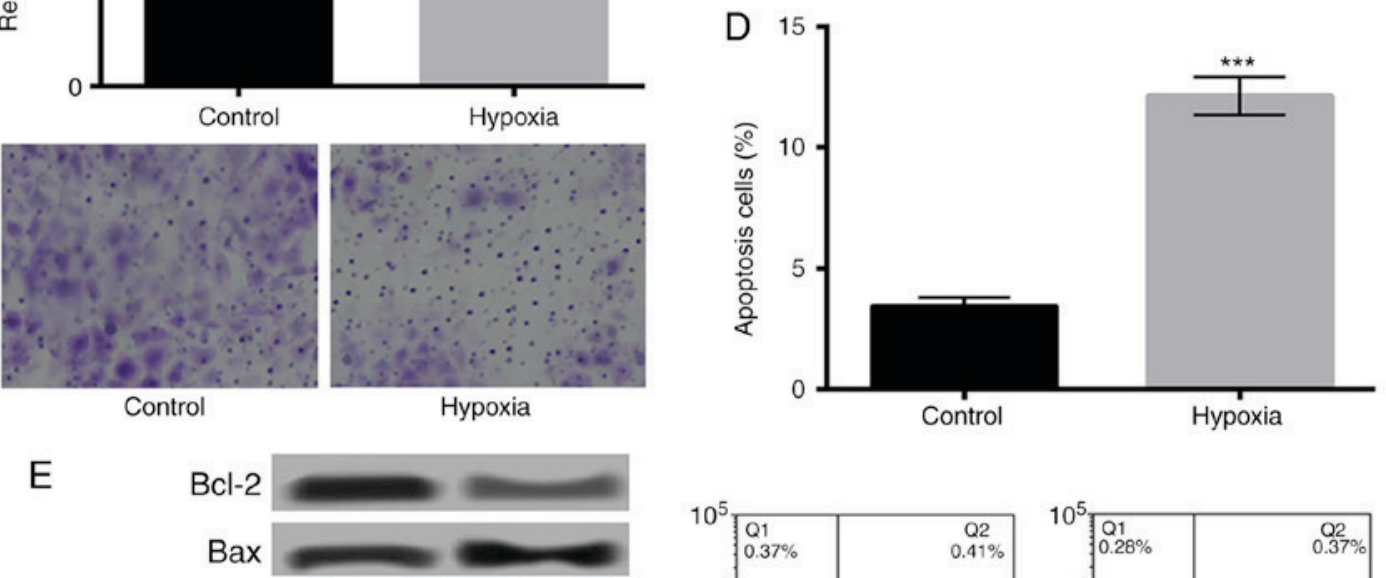

Pro caspase-3
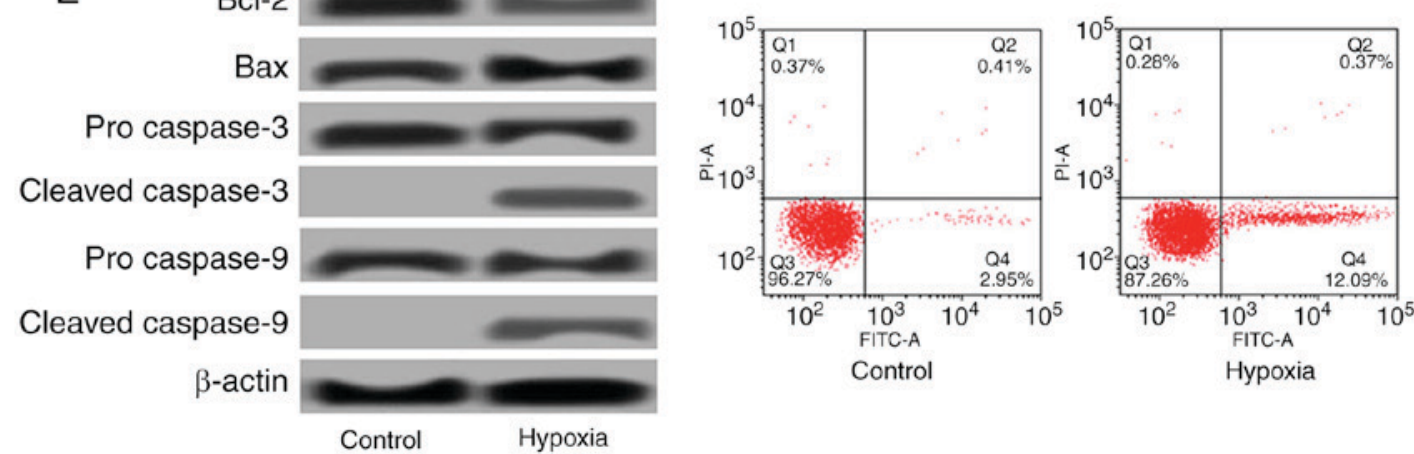

Control

Hypoxia

Figure 1. Hypoxia induced hypoxia injury. Hypoxia significantly decreased the (A) viability, (B) migration and (C) invasion of H9c2 cells. Magnification, x400. (D) Hypoxia significantly increased the apoptosis of $\mathrm{H} 9 \mathrm{c} 2$ cells. (E) The expression levels of apoptosis-associated proteins were markedly different under hypoxic conditions. ${ }^{* *} \mathrm{P}<0.01$ and ${ }^{* * * *} \mathrm{P}<0.001$ vs. control. Bcl-2, B-cell lymphoma 2; Bax, Bcl-2-associated X protein.

changes of cell viability, migration, invasion, and apoptosis. As presented in Fig. 1A-C, hypoxia could significantly decrease the viability, migration, and invasion of $\mathrm{H} 9 \mathrm{c} 2$ cells $(\mathrm{P}<0.01)$. Additionally, Fig. 1D showed that hypoxia significantly increased apoptosis of $\mathrm{H} 9 \mathrm{c} 2$ cells $(\mathrm{P}<0.001)$. The relative expression levels of apoptosis-related proteins changed obviously as well. As shown in Fig. 1E, the expression of Bcl-2 was downregulated, while Bax was upregulated. Moreover, cleaved caspase-3/9 were detected after hypoxia treatment.

Hypoxia promotes the expression of TUG1.The relative expression level of TUG1 under hypoxic condition was detected using RT-qPCR. As shown in Fig. 2, hypoxia treatment significantly increased the expression level of TUG1 $(\mathrm{P}<0.01)$.

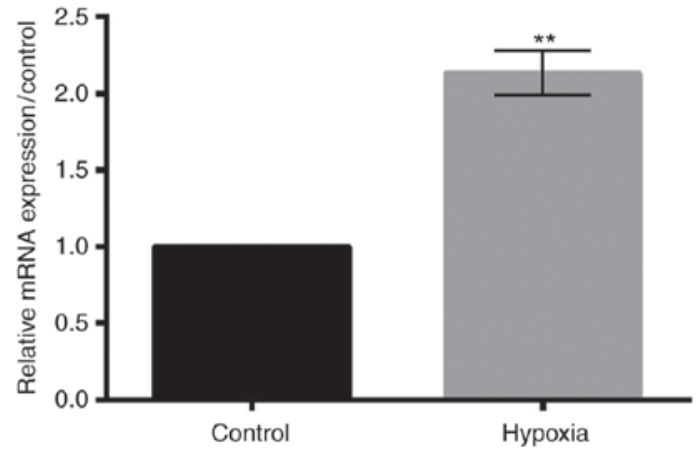

Figure 2. Hypoxia promoted the expression of TUG1. Hypoxia significantly increased the expression level of TUG1. ${ }^{* *} \mathrm{P}<0.01$ vs. the control. TUG1, taurine upregulated 1 (non-protein coding). 

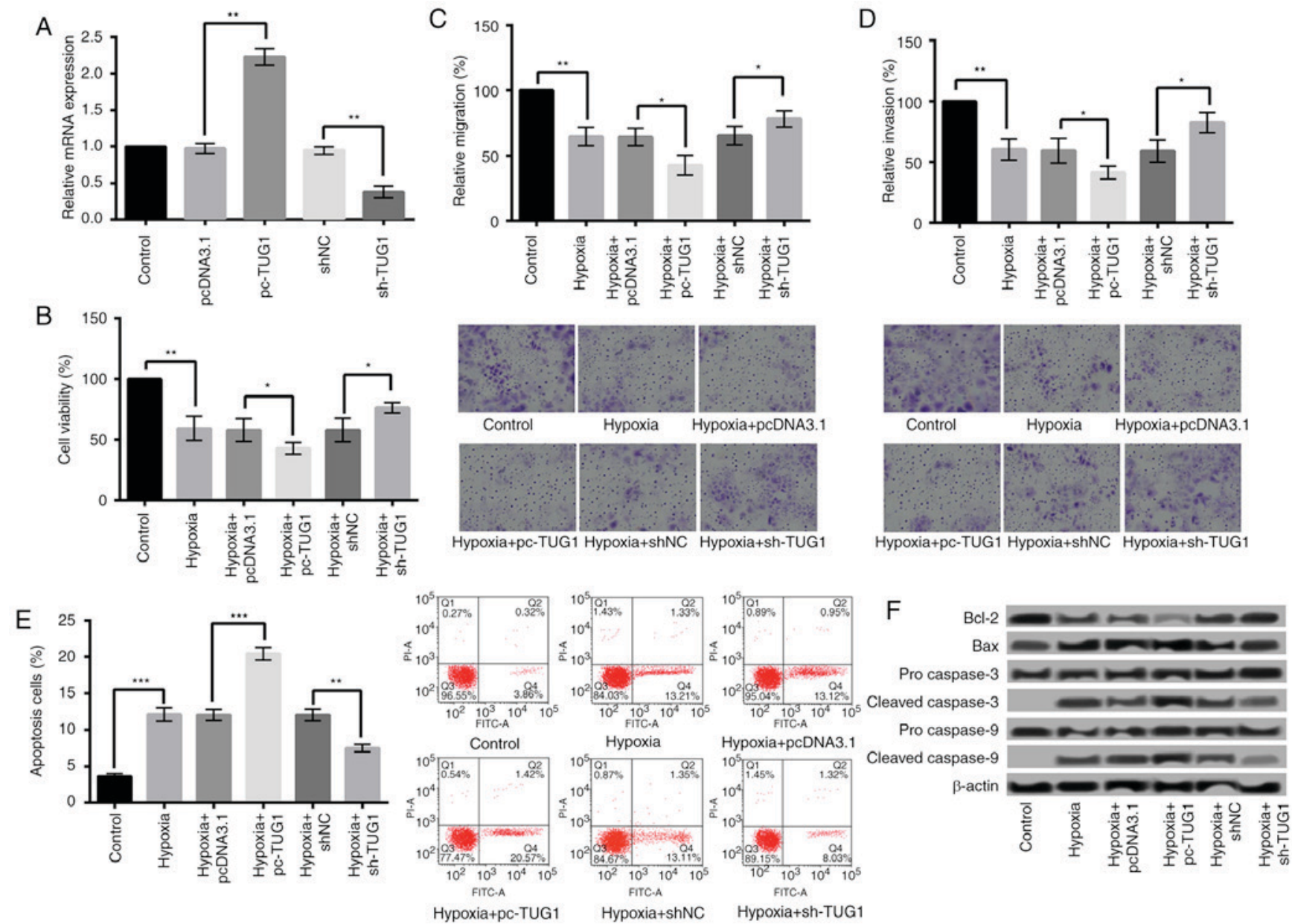

Figure 3. Overexpression of TUG1 aggravated hypoxia-induced injury. (A) TUG1 expression following H9c2 cell transfection with sh-TUG1 and pc-TUG1 Overexpression of TUG1 significantly decreased the (B) viability, (C) migration and (D) invasion, and (E) increased the apoptosis of H9c2 cells when compared with control. Magnification, $\mathrm{x} 400$. (F) The expression levels of apoptosis-associated proteins following TUG1 overexpression. ${ }^{*} \mathrm{P}<0.05$, ${ }^{* * *} \mathrm{P}<0.01$ and ${ }^{* * * *} \mathrm{P}<0.001$, as indicated. TUG1, taurine upregulated 1 (non-protein coding); sh-, small hairpin RNA; pcDNA3.1, plasmid cytomegalovirus promoter DNA3.1; pc-TUG1, TUG1 overexpression group; Bcl-2, B-cell lymphoma 2; Bax, Bcl-2-associated X protein.

Overexpression of TUG1 aggravates hypoxia-induced injury in H9c2 cells, while suppression of TUG1 relieves the injury. To study whether abnormal expression of TUG1 could influence hypoxia-induced injury in H9c2 cells, TUG1 was overexpressed and suppressed in $\mathrm{H} 9 \mathrm{c} 2$ cells. The overexpression or suppression of TUG1 was confirmed by qRT-PCR $(\mathrm{P}<0.01)$ (Fig. 3A). After cell transfection, the effects of hypoxia on cell viability, migration, invasion, and apoptosis of $\mathrm{H} 9 \mathrm{c} 2$ cells were further evaluated. The results showed that compared with pcDNA3.1, overexpression of TUG1 (pc-TUG1) significantly decreased the viability, migration, and invasion (Fig. 3B-D), while increased the apoptosis of $\mathrm{H} 9 \mathrm{c} 2$ cells (Fig. 3E) $(\mathrm{P}<0.05)$. Additionally, the expression of $\mathrm{Bcl}-2$ was further decreased after TUG1 overexpression, while of Bax was further increased. Moreover, the expression levels of cleaved caspase- 3 and caspase- 9 were higher in hypoxia+pc-TUG1 than that in hypoxia+pcDNA3.1 (Fig. 3F). On the contrary, reverse results were obtained when the TUG1 expression was suppressed (Fig. 3B-F).

TUG1 negatively regulates the expression of miR-145-5p and overexpression of TUG1 aggravates hypoxia injury by downregulation of miR-145-5p. Further study found that TUG1 could negatively regulate the expression of
miR-145-5p. The relative expression of TUG1 after cell transfection was shown in Fig. 4A. Fig. 4B presented the expression level of miR-145-5p after H9c2 cells were transfected with miR-145-5p mimic or inhibitor. Additionally, the effect of miR-145-5p overexpression on hypoxia-induced cardiomyocyte injury was detected. As shown in Fig. 4C-F, compared with hypoxia+pc-TUG1+mimic NC group, hypoxia+pc-TUG1+miR-145-5p mimic could relieve hypoxia injury by significantly increasing cell viability, migration, and invasion $(\mathrm{P}<0.05)$, and decreasing apoptosis $(\mathrm{P}<0.001)$. In addition, compared with hypoxia+pc-TUG1+mimic NC group, miR-145-5p overexpression increased Bcl-2 expression, and decreased the expression levels of Bax, cleaved caspase-3 and caspase-9 (Fig. 4G).

miR-145-5p negatively regulates Bnip 3 expression and Bnip3 is a target of miR-145-5p. Study has reported that Bnip3 plays a key role in apoptosis, necrosis and autophagy of cardiomyocytes (15-18). Based on the public miRNA database, we found that the 3'-UTR of Bnip3 was a potential binding site of miR-145-5p, suggesting that Bnip3 may be a direct target of miR-145-5p in cardiomyocytes (Fig. 5A). Then we performed luciferase reporter assay to verify 

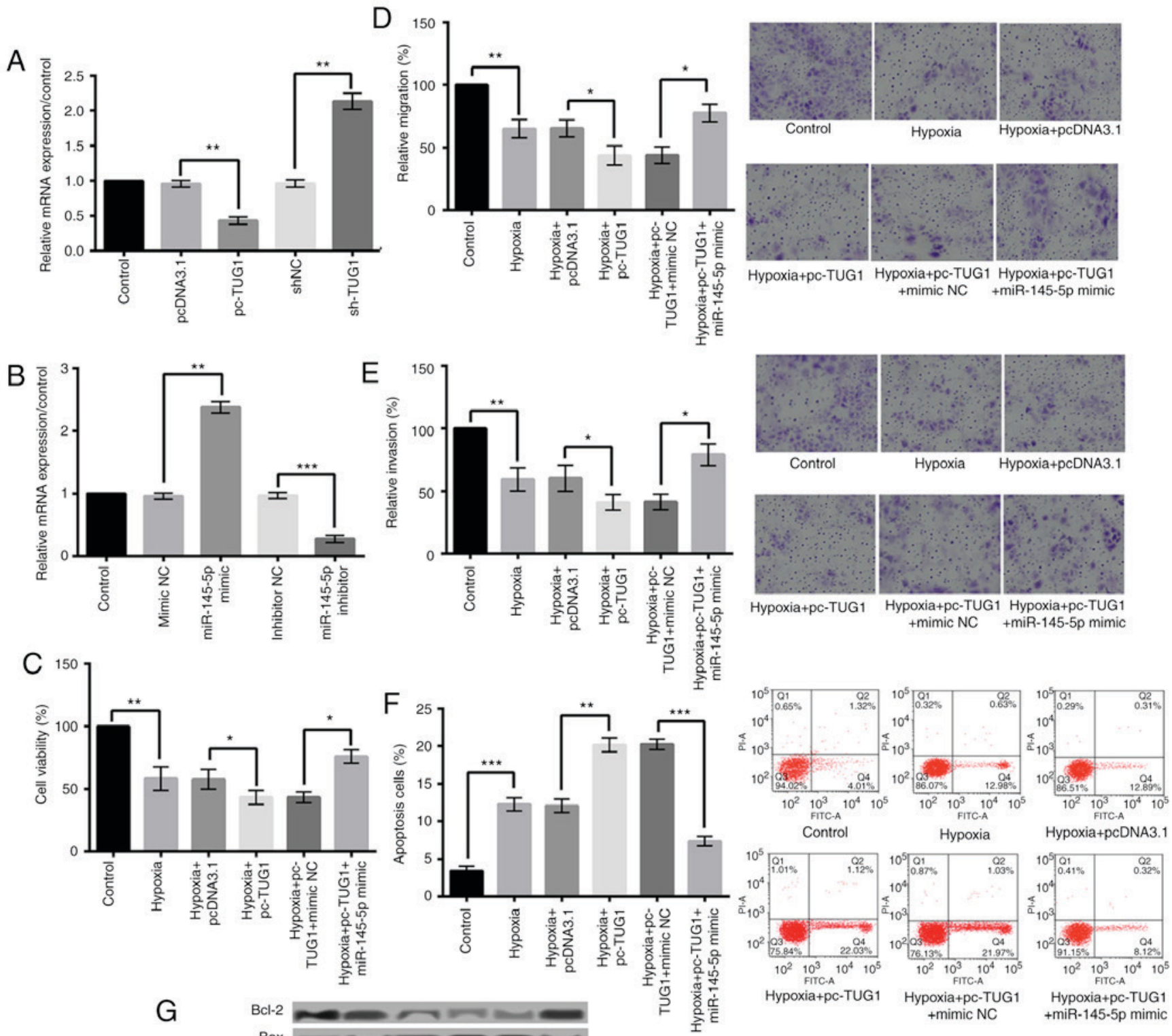

Hypoxia+pcDNA3.
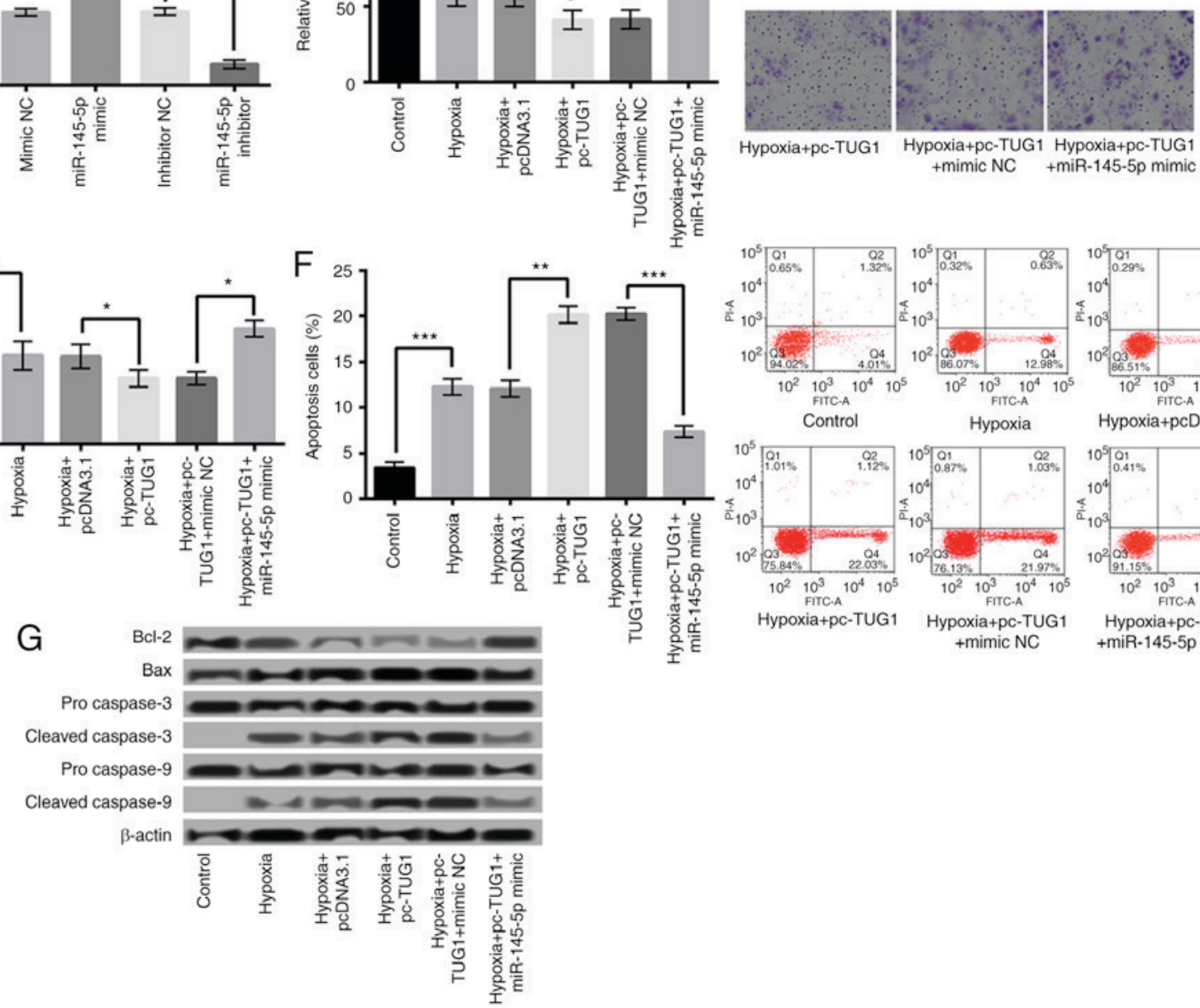

+mimic NC

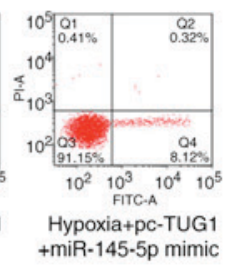

Figure 4. Overexpression of TUG1 aggravated hypoxic injury via downregulation of miR-145-5p. (A) The relative expression of TUG1 following cell transfection. (B) The expression level of miR-145-5p following H9c2 cell transfection with miR-145-5p mimic or inhibitor. Hypoxia+pc-TUG1+miR-145-5p mimic significantly increased the (C) viability, (D) migration and (E) invasion, and decreased (F) the apoptosis of H9c2 cells when compared with the hypoxia+pc-TUG1+mimic NC group. Magnification, $x 400$. (G) miR-145-5p overexpression under hypoxic conditions increased Bcl-2 expression, and decreased the expression levels of Bax, and cleaved caspase-3 and caspase-9. ${ }^{*} \mathrm{P}<0.05,{ }^{* *} \mathrm{P}<0.01$ and ${ }^{* * * *} \mathrm{P}<0.001$, as indicated. TUG1, taurine upregulated 1 (non-protein coding); miR, microRNA; sh-, small hairpin RNA; pcDNA3.1, plasmid cytomegalovirus promoter DNA3.1; pc-TUG1, TUG1 overexpression group; NC, negative control; Bcl-2, B-cell lymphoma 2; Bax, Bcl-2-associated X protein.

whether Bnip3 was a direct target of miR-145-5p. As shown in Fig. 5B, miR-145 overexpression significantly reduced the activity of luciferease gene fused with the Bnip3 wt-3'-UTR $(\mathrm{P}<0.05)$. However, overexpression of miR-145 barely influenced the activity of luciferase gene fused with the Bnip3
3'-UTR mutant. Furthermore, qRT-PCR and western blot analyses found that the relative expression level of Bnip3 was remarkably inhibited by overexpressed miR-145-5p and was raised by suppressed miR-145-5p ( $<<0.01)$ (Fig. 5C and D). These results suggested that Bnip3 was a direct target of 
A

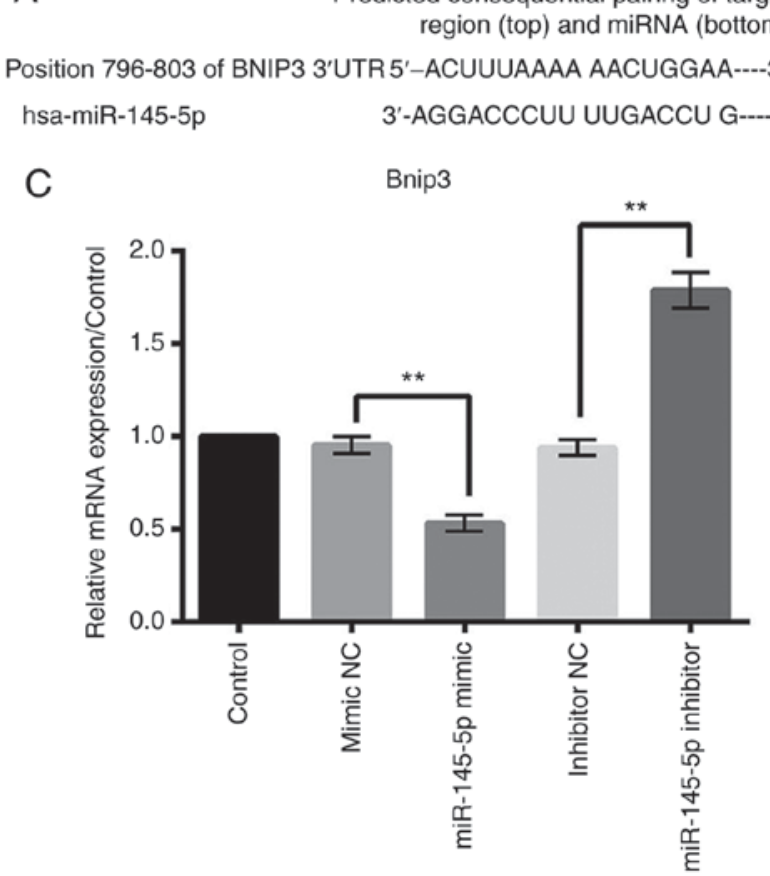

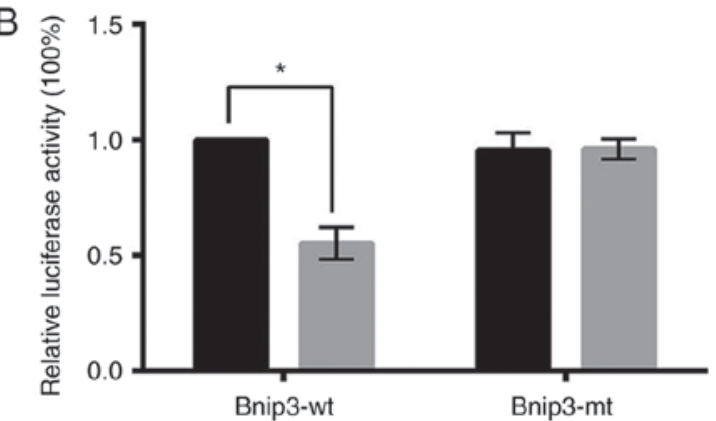

D

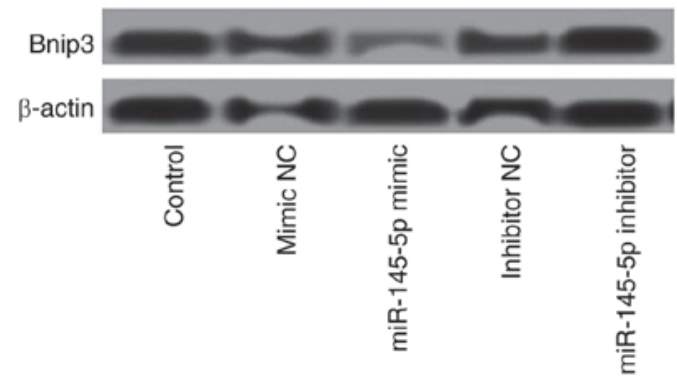

Figure 5. Bnip3 was a target of miR-145-5p. (A) The 3'-UTR of Bnip3 was a potential binding site of miR-145-5p. (B) miR-145-5p overexpression significantly reduced the activity of the luciferase gene fused with the Bnip3 wt-3'-UTR, and had no influence on the activity of the luciferase gene fused with the Bnip3 3'-UTR mutant. The relative (C) mRNA and (D) protein expression level of Bnip3 was markedly inhibited by overexpression of miR-145-5p and was increased with the suppression of miR-145-5p. ${ }^{*} \mathrm{P}<0.05$ and ${ }^{* *} \mathrm{P}<0.01$, as indicated. Bnip3, B-cell lymphoma 2 interacting protein 3 ; miR, microRNA; UTR, untranslated region; wt, wild type; $\mathrm{mt}$, mutant; $\mathrm{NC}$, negative control.

miR-145-5p in cardiomyocytes, and was negatively regulated by miR-145-5p.

Overexpression of miR-145-5p protects against hypoxia-induced injury by downregulation of Bnip3. To further demonstrate that the protective effects of miR-145-5p on cardiomyocytes were achieved by negatively regulating Bnip3, we investigated the effects of Bnip3 overexpression on hypoxia-induced injury. qRT-PCR and western blot were performed to detect the Bnip3 levels after cell transfection. As shown in Fig. 6A and B, transfection of pEX-Bnip3 significantly increased the Bnip3 expression, while transfection of si-Bnip3 significantly suppressed the Bnip3 expression $(\mathrm{P}<0.001)$. Subsequent experiments revealed that the protective effects of miR-145-5p overxpression on cardiomyocytes were abrogated by overexpressed Bnip3, showing that overexpressed Bnip3 significantly reduced the viability, migration, and invasion, and increased the apoptosis of $\mathrm{H} 9 \mathrm{c} 2$ cells (Fig. 6C-F) $(\mathrm{P}<0.01)$. Additionally, the expression of $\mathrm{Bcl}-2$ was decreased, while of Bax, cleaved caspase-3 and caspase-9 was increased (Fig. 6G).

Overexpression of Bnip3 aggravates hypoxia-induced injury via Wnt/ $\beta$-catenin signaling pathways. To explore the underlying mechanisms of Bnip3 overexpression aggravating hypoxia-induced injury, we investigated the effect of Bnip3 overexpression on $\mathrm{Wnt} / \beta$-catenin signaling pathways. As presented in Fig. $6 \mathrm{H}$, Bnip3 overexpression significantly increased the protein expressions of $\mathrm{Wnt} / \beta$-catenin signaling pathways-related proteins, including $\mathrm{Wnt} 3 \mathrm{a} / 5 \mathrm{a}$ and $\beta$-catenin. These results suggested that overexpressed Bnip3 aggravated hypoxia-induced cell injury by activating $\mathrm{Wnt} / \beta$-catenin pathways in $\mathrm{H} 9 \mathrm{c} 2$ cells.

\section{Discussion}

In this study, the effects of TUG1 on the cell hypoxia injury in $\mathrm{H} 9 \mathrm{c} 2$ cells were studied. The results showed that hypoxia induced injury in $\mathrm{H} 9 \mathrm{c} 2$ cells, including inhibiting cell viability, migration and invasion and promoting cell apoptosis. Overexpression of TUG1 aggravated hypoxia injury in H9c2 cells. Further studies showed that miR-145-5p was negatively regulated by TUG1, and TUG1 overexpression aggravated hypoxia injury by downregulation of miR-145-5p. Moreover, we found that miR-145-5p negatively regulated Bnip3 expression and Bnip3 was suggested to be a target gene of miR-145-5p. Overexpression of Bnip3 aggravated hypoxia-induced cell injury by activating Wnt/ $\beta$-catenin pathways in $\mathrm{H} 9 \mathrm{c} 2$ cells. Our study may provide a new strategy for the treatment of myocardial damage induced by hypoxia.

TUG1 was originally found in taurine-treated mouse retinal cells. It has been revealed that TUG1 knockdown leads to malformed outer segments of transfected photoreceptors via increased apoptosis in the newborn retina (19). Additionally, down-regulation of TUG1 has also been suggested to promote apoptosis in many cancer cells $(14,20,21)$. These studies may suggest the critical role of TUG1 in apoptosis. Interestingly, extensive investigation associated with cardiomyocyte ischemic injury found that apoptosis was associated with lots of forms of cardiac pathology, including myocardial ischemia $(22,23)$. Notably, TUG1 is found to function as a miRNA sponge to promote neurons apoptosis under ischemia, which 

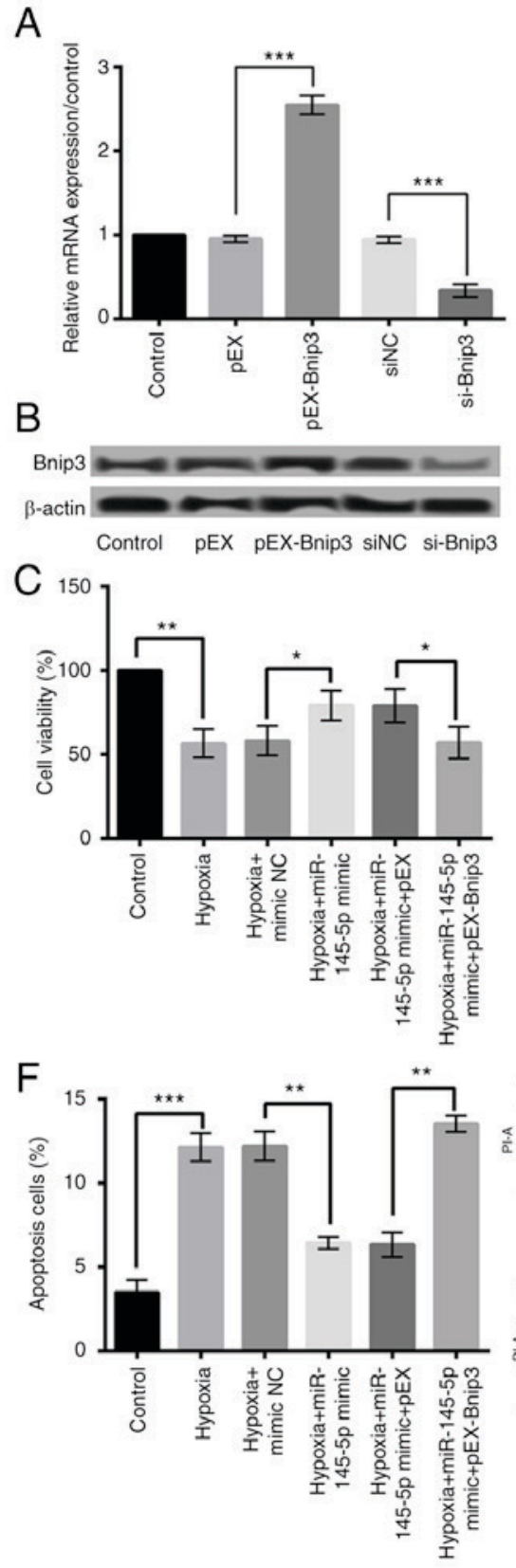

E
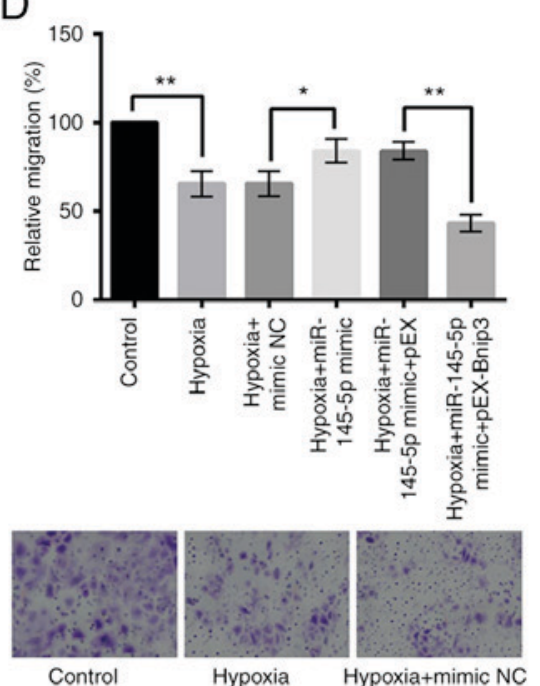

Control

Hypoxia

ypoxia+mimic NC

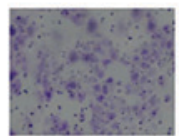

Hypoxia+ miR-145-5p mimic

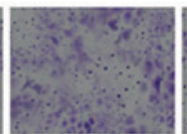

Hypoxia+miRHypoxia+ 145-5p mimic miR-145-5p mimic $+p E X+p E X$ + pEX-Bnip3
+ pEX-Bnip3



\section{G}


Hypoxia+

Hypoxia+miR- Hypoxia+

mimic $+p E X+p E X-B n i p 3$

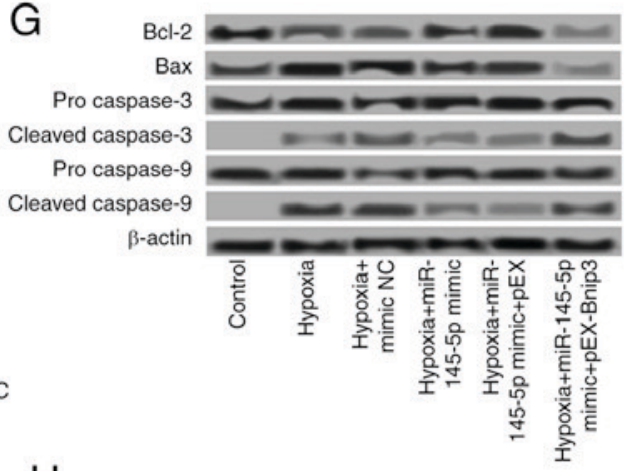

$\mathrm{H}$

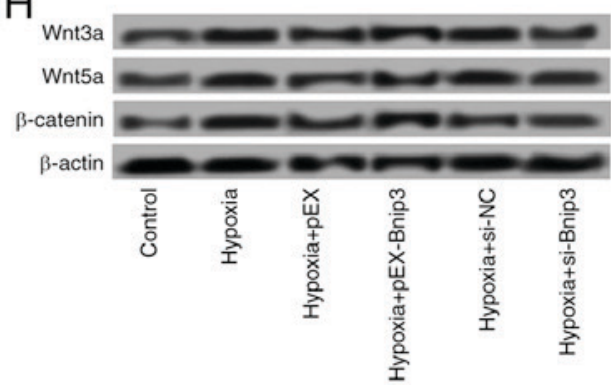

Figure 6. Overexpression of miR-145-5p was protective against hypoxia-induced injury via the downregulation of Bnip3, and the overexpression of Bnip3 aggravated hypoxia-induced injury via the Wnt/ $\beta$-catenin signaling pathway. The relative (A) mRNA and (B) protein expression of Bnip3 following cell transfection. Overexpressed Bnip3 significantly reduced the (C) viability, (D) migration and (E) invasion, and increased the (F) apoptosis of H9c2 cells. Magnification, x400. (G) The expression of Bcl-2 was decreased, while that of Bax, and cleaved caspase-3 and caspase-9 was increased following Bnip3 overexpression. (H) Bnip3 overexpression increased the protein expressions of proteins associated with the Wnt/3-catenin signaling pathways, including Wnt3a/5a and $\beta$-catenin. ${ }^{*} \mathrm{P}<0.05,{ }^{* *} \mathrm{P}<0.01$ and ${ }^{* * *} \mathrm{P}<0.001$, as indicated. Bnip3, B-cell lymphoma 2 interacting protein 3; miR, microRNA; si-, small interfering RNA.

possibly severed as a new therapeutic target in stroke (24). In this study, overexpression of TUG1 was found to significantly decreased the viability, migration, and invasion, and increased the apoptosis of hypoxia-induced H9c2 cells, suggesting the important role of TUG1 in myocardial ischemia.

MiR-145 is a tumor suppressor miRNA which suppresses proliferation and induce apoptosis in various tumor cell lines $(25,26)$. It is reported that TUG1 can influence epithelial-to-mesenchymal transition in several cancers through targeting miR-145 $(20,27)$. Serum miR-145 is found positively correlated with plasma high-sensitivity C-reactive protein (hs-CRP) and the combination of hs-CRP and serum miR-145 may be an effective approach for predicting acute ischemia stroke (28). Recently, the functions of miR-145 in heart were explored. In the study of $\mathrm{Li}$ et al (29), miR-145 was suggested to exert a protective effect against the oxidative stress-induced 
apoptosis in cardiomyocytes. Importantly, they have demonstrated that under oxidative stress, miR-145 protects against the mitochondria apoptotic pathway activation in cardiomyocytes via targeting Bnip3 directly. In accordance with their study, our results showed that TUG1 overexpression aggravated hypoxia injury by downregulation of miR-145-5p. Moreover, Bnip3 was a target of miR-145-5p and was negatively regulated by miR-145-5p.

Bnip3, primarily in the mitochondrial outer membrane, belongs to $\mathrm{BH} 3$-only subfamily of $\mathrm{Bcl}-2$ family proteins, which antagonizes the activity of pro-survival proteins and promotes apoptosis $(30,31)$. Normally, the Bnip3 expression is undetectable in most organs, including the heart. However, its expression level can be increased by hypoxia (32). During myocardial ischemia and reperfusion, Bnip3 is found to act as a mitochondrial sensor of oxidative stress (16). Graham et al (33) also reported that Bnip3 is overexpressed in heart following acute ischemia, and in chronic heart failure after myocardial infarction. Interestingly, in this study, overexpression of Bnip3 was found to aggravate hypoxia-induced cell injury, which was in consistence with the studies above. Furthermore, the overexpressed Bnip3 aggravating hypoxia-induced cell injury was found to be achieved by activating $\mathrm{Wnt} / \beta$-catenin pathways.

It is reported that interacting cells can form a "cellular interactome' under a defined condition. After cardiac injury, different populations of cells in the heart can construct a complex cardiac cellular interactome, which is regulated by many signaling systems (34). The Wnt/ $\beta$-catenin signaling pathway has been demonstrated to play a critical role in cardiac development, and in orchestrating a cardiac injury response $(35,36)$. Therefore, our study further suggested the role of $\mathrm{Wnt} / \beta$-catenin signaling pathway in hypoxia-induced injury of cardiomyocytes.

In conclusion, our data suggest that TUG1 overexpression aggravates hypoxia injury of cardiomyocytes by regulating miR-145-5p-Bnip3 axis to activate $\mathrm{Wnt} / \beta$-catenin pathways. Therefore, TUG1 may be used as a diagnostic marker and therapeutic target in myocardial ischemia. However, there is lack of in vivo research in myocardial ischemia to better investigate the role of LncRNA TUG1 in the whole organism. Further clinical and in vivo studies are still needed to confirm the results.

\section{References}

1. Reimer KA and Ideker RE: Myocardial ischemia and infarction: Anatomic and biochemical substrates for ischemic cell death and ventricular arrhythmias. Human Pathol 18: 462-475, 1987.

2. Buja LM, Hagler HK and Willerson JT: Altered calcium homeostasis in the pathogenesis of myocardial ischemic and hypoxic injury. Cell Calcium 9: 205-217, 1988.

3. Farber JL, Chien KR and Mittnacht S Jr: Myocardial ischemia: The pathogenesis of irreversible cell injury in ischemia. Am J Pathol 102: 271-281, 1981.

4. Buja LM: Myocardial ischemia and reperfusion injury: Cardiovasc Pathol 14: 170-175, 2005.

5. American College of Emergency Physicians; Society for Cardiovascular Angiography and Interventions; O'Gara PT, Kushner FG, Ascheim DD, Casey DE Jr, Chung MK, de Lemos JA, Ettinger SM, Fang JC, et al: 2013 ACCF/AHA guideline for the management of ST-elevation myocardial infarction: Executive summary: A report of the american college of cardiology foundation/american heart association task force on practice guidelines. J Am Coll Cardiol 61: 485-510, 2013.
6. Gartler SM and Riggs AD: Mammalian X-chromosome inactivation. Annu Rev Genet 17: 155-190, 1983.

7. Shi X, Sun M, Liu H, Yao Y and Song Y: Long non-coding RNAs: A new frontier in the study of human diseases. Cancer Lett 339: 159-166, 2013.

8. Wapinski $\mathrm{O}$ and Chang HY: Long noncoding RNAs and human disease. Trends Cell Biol 21: 354-361, 2011.

9. Li J, Xuan Z and Liu C: Long non-coding RNAs and complex human diseases. Int J Mol Sci 14: 18790-18808, 2013.

10. Grote P, Wittler L, Hendrix D, Koch F, Währisch S, Beisaw A, Macura K, Bläss G, Kellis M, Werber M and Herrmann BG: The tissue-specific lncRNA Fendrr is an essential regulator of heart and body wall development in the mouse. Dev Cell 24: 206-214, 2013.

11. Ishii N, Ozaki K, Sato H, Mizuno H, Saito S, Takahashi A, Miyamoto Y, Ikegawa S, Kamatani N, Hori M, et al: Identification of a novel non-coding RNA, MIAT, that confers risk of myocardial infarction. J Hum Genet 51: 1087-1099, 2006.

12. Yin DD, Zhang EB, You LH, Wang N, Wang LT, Jin FY, Zhu YN, Cao LH, Yuan QX, De W and Tang W: Downregulation of lncRNA TUG1 affects apoptosis and insulin secretion in mouse pancreatic $\beta$ cells. Cell Physiol Biochem 35: 1892-1904, 2015.

13. Zhang Q, Geng PL, Yin P, Wang XL, Jia JP and Yao J: Down-regulation of long non-coding RNA TUG1 inhibits osteosarcoma cell proliferation and promotes apoptosis. Asian Pac J Cancer Prev 14: 2311-2315, 2013.

14. Han Y, Liu Y, Gui Y and Cai Z: Long intergenic non-coding RNA TUG1 is overexpressed in urothelial carcinoma of the bladder. J Surg Oncol 107: 555-559, 2013.

15. Lomonosova $\mathrm{E}$ and Chinnadurai G: $\mathrm{BH} 3$-only proteins in apoptosis and beyond: An overview. Oncogene 27 (Suppl 1): S2-S19, 2008.

16. Kubli DA, Quinsay MN, Huang C, Lee Y and Gustafsson AB: Bnip3 functions as a mitochondrial sensor of oxidative stress during myocardial ischemia and reperfusion. Am J Physiol Heart Circ Physiol 295: H2025-H2031, 2008.

17. Kubasiak LA, Hernandez OM, Bishopric NH and Webster KA: Hypoxia and acidosis activate cardiac myocyte death through the Bcl-2 family protein BNIP3: Proc Natl Acad Sci USA 99: $12825-12830,2002$.

18. Guo K, Searfoss G, Krolikowski D, Pagnoni M, Franks C, Clark K, Yu KT, Jaye M and Ivashchenko Y: Hypoxia induces the expression of the pro-apoptotic gene BNIP3. Cell Death Differ 8: 367-376, 2001.

19. Young TL, Matsuda T and Cepko CL: The noncoding RNA taurine upregulated gene 1 is required for differentiation of the murine retina. Curr Biol 15: 501-512, 2005.

20. Tan J, Qiu K, Li M and Liang Y: Double-negative feedback loop between long non-coding RNA TUG1 and miR-145 promotes epithelial to mesenchymal transition and radioresistance in human bladder cancer cells. FEBS Lett 589: 3175-3181, 2015.

21. Liu Y, Yang S and Zhang X: WITHDRAWN: Down-regulation of long non-coding RNA TUG1 suppresses melanoma cell proliferation and induces apoptosis via up-regulating microRNA-9. Biochem Biophys Res Commun, 2013.

22. Buja LM: Modulation of the myocardial response to ischemia. Lab Invest 78: 1345-1373, 1998.

23. Buja LM and Entman ML: Modes of myocardial cell injury and cell death in ischemic heart disease. Circulation 98: 1355-1357, 1998.

24. Chen S, Wang M, Yang H, Mao L, He Q, Jin H, Ye ZM, Luo XY, $\mathrm{Xia}$ YP and $\mathrm{Hu}$ B: LncRNA TUG1 sponges microRNA-9 to promote neurons apoptosis by up-regulated $\mathrm{Bcl} 2111$ under ischemia. Biochem Biophys Res Commun 485: 167-173, 2017.

25. Spizzo R, Nicoloso MS, Lupini L, Lu Y, Fogarty J, Rossi S, Zagatti B, Fabbri M, Veronese A, Liu X, et al: miR-145 participates with TP53 in a death-promoting regulatory loop and targets estrogen receptor-alpha in human breast cancer cells. Cell Death Differ 17: 246-254, 2010.

26. Xu Q, Liu LZ, Qian X, Chen Q, Jiang Y, Li D, Lai L and Jiang BH: MiR-145 directly targets p70S6K1 in cancer cells to inhibit tumor growth and angiogenesis. Nucleic Acids Res 40: 761-774, 2012.

27. Lei H, Gao Y and Xu X: LncRNA TUG1 influences papillary thyroid cancer cell proliferation, migration and EMT formation through targeting miR-145: Acta Biochim Biophys Sin (Shsnghai) 49: 588-597, 2017.

28. Jia L, Hao F, Wang W and Qu Y: Circulating miR-145 is associated with plasma high-sensitivity C-reactive protein in acute ischemic stroke patients. Cell Biochem Funct 33: 314-319, 2015 . 
29. Li R, Yan G, Li Q, Sun H, Hu Y, Sun J and Xu B: MicroRNA-145 protects cardiomyocytes against hydrogen peroxide (H2O2)-induced apoptosis through targeting the mitochondria apoptotic pathway. PLoS One 7: e44907, 2012.

30. Ray R, Chen G, Vande Velde C, Cizeau J, Park JH, Reed JC, Gietz RD and Greenberg AH: BNIP3 heterodimerizes with $\mathrm{Bcl}-2 / \mathrm{Bcl}-\mathrm{X}(\mathrm{L})$ and induces cell death independent of a Bcl-2 homology 3 (BH3) domain at both mitochondrial and nonmitochondrial sites. J Biol Chem 275: 1439-1448, 2000.

31. Yasuda M, Theodorakis P, Subramanian T and Chinnadurai G: Adenovirus E1B-19K/BCL-2 interacting protein BNIP3 contains a BH3 domain and a mitochondrial targeting sequence. J Biol Chem 273: 12415-12421, 1998.

32. Bruick RK: Expression of the gene encoding the proapoptotic Nip3 protein is induced by hypoxia. Proc Natl Acad Sci USA 97: 9082-9087, 2000.
33. Graham RM, Frazier DP, Thompson JW, Haliko S, Li H, Wasserlauf BJ, Spiga MG, Bishopric NH and Webster KA: A unique pathway of cardiac myocyte death caused by hypoxia-acidosis. J Exp Biol 207: 3189-3200, 2004.

34. Deb A: Cell-cell interaction in the heart via Wnt/ $\beta$-catenin pathway after cardiac injury. Cardiovasc Res 102: 214-223, 2014.

35. Bergmann MW: WNT signaling in adult cardiac hypertrophy and remodeling: Lessons learned from cardiac development. Circ Res 107: 1198-1208, 2010.

36. Gessert $\mathrm{S}$ and Kühl M: The multiple phases and faces of wnt signaling during cardiac differentiation and development. Circ Res 107: 186-199, 2010.

This work is licensed under a Creative Commons Attribution-NonCommercial-NoDerivatives 4.0 International (CC BY-NC-ND 4.0) License. 